\title{
New Results on the Genetic Cryptanalysis of TEA and Reduced-round Versions of XTEA
}

\author{
Julio César HERNÁNDEZ CASTRO \\ LIFL-CNRS \\ 59655 Villeneuve d'Ascq, France \\ hernande@lifl.fr \\ Pedro ISASI VIÑUELA \\ Computer Science Department \\ Carlos III University \\ 28911 Leganés, Madrid, Spain \\ isasi@inf.uc3m.es
}

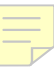

Received 31 March 2004

Revised manuscript received 2 July 2004

\begin{abstract}
Recently, a quick and simple way of creating very efficient distinguishers for cryptographic primitives such as block ciphers or hash functions, was presented and proved useful by the authors. In this paper, this cryptanalytic attack (named genetic cryptanalysis after its use of genetic algorithms) is shown to be successful when applied over reduced-round versions of the block cipher XTEA. Efficient distinguishers for XTEA are proposed for up to 4 cycles ( 8 rounds). Additionally, a variant of this genetic attack is also introduced, and their results over the block cipher TEA presented, which are the most powerful published to date.
\end{abstract}

Keywords: Cryptanalysis, Distinguishers, Block Ciphers, XTEA, TEA.

\section{$\S 1$ Introduction}

The construction of a distinguisher ${ }^{2)}$ (i.e. an algorithm that is capable of distinguishing a random permutation or random mapping from a given cryptographic primitive such as a block cipher or hash function) is one of the objectives of any cryptanalyst.

Although a distinguisher may or may not be used to recover some of the plaintext or key bits, the existence of an efficient and effective distinguisher always means the cryptographic primitive in question is weak ${ }^{2.3)}$ and must be discarded for any cryptographic usage. 


\subsection{The Block Cipher XTEA}

XTEA stands for eXtended Tiny Encryption Algorithm. It is the name of an improvement over TEA, a previous block cipher also invented by David Wheeler and Roger Needham, members of the Computer Security Laboratory of Cambridge University.

The original TEA block cipher was presented in the 1994 Fast Software Encryption Workshop, ${ }^{4)}$ but an related-key attack was proposed in Reference ${ }^{5)}$ and the new XTEA algorithm was the answer of TEA developers's to avoid this attack, although they argued it had little to none practical implications.

XTEA, as TEA, is a very fast block cipher that does not use predefined tables or S-boxes and does not need much initialisation time. It is a Feistel type algorithm. It works over 64 bit blocks and uses keys of 128 bits. The authors conjectured it had a security (with 8,16 or 32 rounds) comparable with the the Data Encryption Standard (DES), being quite faster (at least with 8 rounds). However, in the light of some recent results ${ }^{5,6)}$ this assertion seems to be extremely optimistic. It is very portable, simple and efficient as its compact code shows:

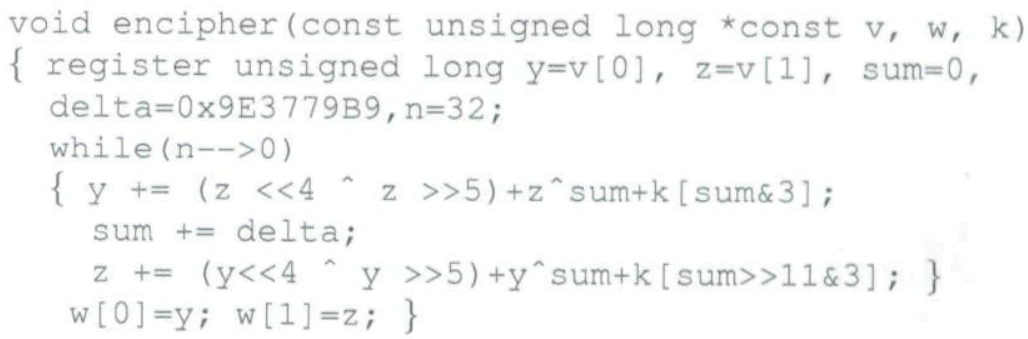

\subsection{Overview of Our Methodology}

Our method, already presented in Reference, ${ }^{1)}$ is based in the search for subsets of the input space that produce a high (statistically significant) deviation of the distribution of a given subset of the output produced by a given cryptographic primitive. For this search we use a genetic-algorithm based approach in which individuals of the population codify bit masks that are used to perform a logical AND with randomly generated inputs. In this way, we get an extremely efficient representation of those input subsets characterized by having some of the input bits fixed to a zero value. Input subsets of this form are evaluated performing chi-square tests over the output distribution of the subset under observation. These tests vary, because additional rounds of XTEA exponentially increase the dispersion of the output, and thus the difficulty of finding significant deviations. The genetic algorithm will evolve individuals and populations towards those that, by fixing the input bits that have a greater effect over the observed output, produce a higher deviation from the expected probability distribution. 


\section{$\S 2$ Results for XTEA}

Below we present the results of our attempts to evolve distinguishers for reduced round variants of XTEA. We start with the simplest case (one cycle of XTEA) and progressively increase the number of rounds.

\subsection{One Cycle XTEA}

Every input subset (or bitmask) is tested by generating $2^{11}$ random inputs, and then performing a logical AND between each of these inputs and the bitmask. Then XTEA1 (i.e. one cycle of XTEA) is applied over thus generated vectors, and the values of the least significant eight bits of the first output word of XTEA, that is w $[0] \& 255$, are computed.

We have focused in this particular part of the output because there are authors, notably, ${ }^{7)}$ that have shown that block ciphers using rotation as one of their round operations (as is the case of XTEA) tend to exhibit bad distributions in their least significant bits of their output words. The fitness function we propose for the genetic algorithm is highly related with the chi-square statistic $\chi^{2}$ which in our case measures the deviation of the observed output distribution from uniform, in this way:

$$
\text { If } \chi^{2}=522480 \text { then fitness }=w^{4} \text { else fitness }=\chi^{2}
$$

The idea behind this fitness function is that the value of the chi square statistic cannot increase indefinitely, but has a maximum. The maximum value of the chi square statistic corresponding to a distribution with 255 degrees of freedom and $2^{11}$ observations is precisely 522480 , which is obtained when all the possible 256 outputs collapse into a single one. Once we find bitmasks that produce this maximum deviation, our search must continue by looking for those bitmasks that are heavier (have more 1's), and this is the reason of including the weight $\mathrm{w}$ in the formula above, once the maximum deviation is obtained. Heavier bitmasks are preferred because they allow for a larger set of different inputs, so in this sense we can say they are more general, and also give more information about the input subset (the 0's in the bitmask or inactive bits) that has a stronger influence over the observed output. Obviously, we must try to maximise this fitness value. The code we used for testing was the implementation of the genetic algorithm of William M. Spears, from the Navy Center for Applied Research. Other parameters needed for running the genetic algorithm are the size of the population, the mutation rate and the crossover probability. Those values were fixed, respectively, to $100,0.005$ and 0.85 after some trial and error testing that showed they produced good results. The best bitmask we found after around 730 generations and 34000 evaluations for the fitness function presented above was $m_{1}$ :

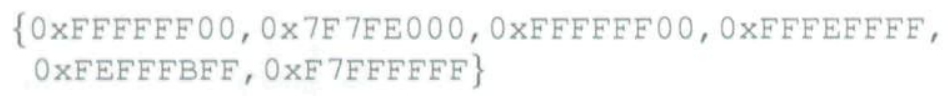

which has a weight of 157 and produces a chi square of 522240 . 
It was then tested with different, previously unseen input subsets of size $2^{11}$ and in every case it produced maximal deviations (i.e. a collapse of the output). This bitmask can be used to construct an exceptionally efficient and simple distinguisher for XTEA1, the pseudocode of which is presented below:

INPUT: F: $Z_{2}^{192} \longmapsto Z_{2}^{64}$, a random mapping or XTEA1 ALGORITHM:

- Generate a random vector $v \in Z_{2}^{192}$

- Apply the mask $m_{1}$, getting $v^{\prime}=v \& m_{1}$ which can take any of $2^{157}$ possible values

- Compute $F\left(v^{\prime}\right)=w[0] w[1]$

- Compute $r=w[0] \& 255$

OUTPUT: If $\mathrm{r}=0$ then $\mathrm{F}$ is XTEA1 else $\mathrm{F}$ is not XTEA1

It is interesting to point out that this distinguisher is extremely efficient, given that with only one input text it has a false positive probability of $1 / 256$ (or around 0.4 pct) and a zero probability of false negatives.

\subsection{Two Cycles XTEA}

XTEA with two cycles (XTEA2) is much harder than XTEA1. The additional cycle significantly increases the strength of the algorithm and no usable bitmaks producing maximal deviation (collapses) were found, so the fitness function used for XTEA1 is not adequate here. Although a fitness function consisting simply of the chi-square statistic can break XTEA2, it needs some extra care with technical details (mainly a selection proportional to rank and not to fitness and different mutation and crossover probabilities) to produce good results. Another drawback of this fitness function is that it shows a very low convergence towards good solutions (those which produce results statistically better than can be expected at random). Furthermore, this simplistic approximation is not applicable to XTEA3. So, after solving the case for XTEA3, we turned back to XTEA2 and observed that the same fitness function would be very adequate, so we will present now a new fitness function that reflects an idea that is enough for breaking both XTEA2 and XTEA3. The fitness function used in this case is shown below:

$$
\text { If } \chi^{2} \geq 403.4579 \text { then fitness }=1 / w^{3}+w^{4} \text { else fitness }=1 / w^{3}
$$

The idea behind this fitness function is to divide the search for good and heavy bitmasks in two phases. In the first one, the chi-square values will be around the 0.5 percentile, and the fitness function above will simply look for low-weighted bitmasks.

When the bitmasks are sufficiently low to produce high values of the chi square statistic (above the threshold of 403.4579), then the objective is to find heavier bitmasks between those which produce a very high statistical value (Table 1 shows some p-values of a chi-square distribution with 255 degrees of freedom). 


\section{Table 1}

\begin{tabular}{|c|r|}
\hline probability & $p$-value \\
\hline $1 / 2$ & 254.33 \\
$10^{-2}$ & 310.45 \\
$10^{-3}$ & 330.51 \\
$10^{-4}$ & 347.65 \\
$10^{-5}$ & 362.98 \\
\hline
\end{tabular}

In this way, we do not maximize the chi-square value but the weight of the masks that produce a statistic value over a given threshold. In this case, the threshold is the corresponding value for a chi-square distribution with 255 degrees of freedom and a p-value of $5 \cdot 10^{-9}$, so it clearly shows a very strong deviation from randomness.

Using this approximation, we got the following bitmask $m_{2}$ after around 530 generations and 25000 evaluations:

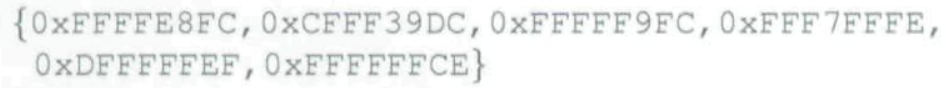

which has a weight of 166 .

After testing this bitmask with other previously unseen inputs, the average chi square statistic obtained was 442.4. It is then feasible to construct an efficient distinguisher for XTEA2, as shown in the following pseudocode:

INPUT: F: $Z_{2}^{192} \longmapsto Z_{2}^{64}$, a random mapping or XTEA2

\section{ALGORITHM:}

- Generate $2^{11}$ random vectors $v_{i} \in Z_{2}^{192}$

- Apply mask $m_{2}$ to every vector $v_{i}$, getting $v_{i}^{\prime}=v_{i} \& m_{2}$ that can take any of $2^{166}$ possible values

- Compute $F\left(v_{i}^{\prime}\right)=w[0]_{i} w[1]_{i}$

- Compute $r_{i}=w[0]_{i} \& 255$

- Perform a chi-square test for checking if the observed distribution of $r_{i}$ is consistent with the expected uniform distribution, calculating the corresponding chi-square statistic $\chi^{2}$

OUTPUT: If $\chi^{2}>390.0315$ then $\mathrm{F}$ is TEA2 else $\mathrm{F}$ is not TEA2

The 390.0315 is the value corresponding to a p-value of $10^{-7}$, so the distinguisher described before will have a false positive probability of $10^{-7}$ and a false negative probability even closer to zero.

\subsection{Three Cycles XTEA}

Using essentially the same approximation described before, we get the following bitmask $m_{3}$ for XTEA with 3 cycles after around 1600 generations and 76000 evaluations 
which has a weight of 118 and produces an average chi-square statistic of 530.6 over previously unseen cases.

The distinguisher will be, then

INPUT: F: $Z_{2}^{192} \longmapsto Z_{2}^{64}$, a random mapping or XTEA3 ALGORITHM:

- Generate $2^{11}$ random vectors $v_{i} \in Z_{2}^{192}$

- Apply mask $m_{3}$ to every vector $v_{i}$, getting $v_{i}^{\prime}=v_{i} \& m_{3}$ that can take any of $2^{118}$ possible values

- Compute $F\left(v_{i}^{\prime}\right)=w[0]_{i} w[1]_{i}$

- Compute $r_{i}=w[0]_{i} \& 255$

- Perform a chi-square test for checking if the observed distribution of $r_{i}$ is consistent with the expected uniform distribution, calculating the corresponding chi-square statistic $\chi^{2}$

OUTPUT: If $\chi^{2}>330.5197$ then $\mathrm{F}$ is TEA3 else $\mathrm{F}$ is not TEA3

The 330.5197 threshold is the value corresponding to a p-value of $10^{-3}$, so this distinguisher will have a false positive probability of $10^{-3}$ and a false negative probability of around 0.5

\subsection{Four Cycles XTEA}

This is a considerably harder case. When using the same approximation that was successful over the prior two cases, we only managed to obtain bitmasks of relatively low weight.

This is interesting and can be useful for different cryptanalytic purposes, for example for starting a search of impossible differentials, but it is not enough in this case, as it does not allow for $2^{64}$ different inputs. When not having at least $2^{64}$ different elements in the input subset, we can not ask to obtain a good distribution of the output, as it must somehow reflect the low entropy of the input (although it do not need to be precisely in $w[0] \& 255$, the bits we observe). So we need a different approximation, a subtler one, able of distinguishing from randomness behaviors that may have past undetected by other, less sensible, tests.

Our proposal is based in a test used to measure the Strict Avalanche Criterion or SAC. $\left.{ }^{8}\right)$ The SAC will be measured just by flipping at random a bit that is at a position where there is an active bit (i.e. one that has a 1 in the corresponding input mask), then measuring the Hamming distance of the two outputs.

A mapping $Z_{2}^{m} \longmapsto Z_{2}^{n}$ has the SAC over a certain input subset $S \in Z_{2}^{m}$ iff the Hamming distance of the output of inputs $x$ and $x^{\prime}$ that only differ in a position (i.e. $w\left(x \oplus x^{\prime}\right)=1$ ) and belong to the input subset $\mathrm{S}$ follow a Binomial distribution. In the case of TEA, we should have a $B(1 / 2,64)$. It is 
important to note that the satisfactibily of this criterion implies the avalanche effect (changes in the input of only one bit should produce a change of around half of the output), because the average of the distribution $B(1 / 2, n)$ is $n / 2$. For testing if a given bitmask represents an input subset which elements meet the SAC, we propose to perform a chi-square test for the goodness of fit of the observed output distribution of the Hamming values with respect to the theoretical Binomial distribution. In this case, we have a chi square statistic with 64 degrees of freedom. Table 2 shows some p-values of this distribution.

Table 2

\begin{tabular}{|c|r|}
\hline probability & p-value \\
\hline $1 / 2$ & 63.33 \\
$10^{-2}$ & 93.21 \\
$10^{-3}$ & 104.71 \\
$10^{-4}$ & 114.83 \\
$10^{-5}$ & 124.10 \\
\hline
\end{tabular}

Using this approximation, we got the following bitmask $m_{4}$ :

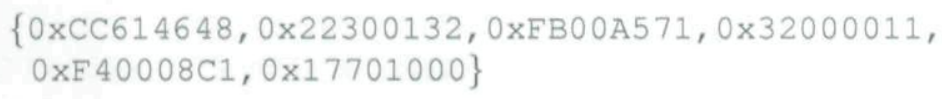

with a weight of 57 (the weight is not a limit here, as far as sufficiently many input elements exist to perform the test), a fitness of 313.06 and an average chisquare value over previously unseen examples of 278.87 . In this case, we did not introduced any preference for heavier bitmasks. The corresponding distinguisher pseudocode will then be:

INPUT: F: $Z_{2}^{192} \longmapsto Z_{2}^{64}$, a random mapping or XTEA4 ALGORITHM:

- Generate $2^{11}$ random vectors $v_{i} \in Z_{2}^{192}$

- Apply mask $m_{4}$ to every vector $v_{i}$, getting $v_{i}^{\prime}=v_{i} \& m_{4}$ that can take any of $2^{57}$ possible values

- For every $v_{i}^{\prime}$

- Select a position p at random from those that have a 1 in the bitmask (active positions)

- Generate $v_{i}^{\prime \prime}$ by changing the value of $v_{i}^{\prime}$ at position $\mathrm{p}$ End For

- Compute $r_{i}=H\left(F\left(v_{i}^{\prime}\right), F\left(v_{i}^{\prime \prime}\right)\right)$, the Hamming distance between $F\left(v_{i}^{\prime}\right)$ and $F\left(v_{i}^{\prime \prime}\right)$

- Perform a chi-square test for checking if the observed distribution of $r_{i}$ is consistent with the expected distribution, calculating the corresponding chi-square statistic $\chi^{2}$

OUTPUT: If $\chi^{2}>148.3564$ then $\mathrm{F}$ is XTEA4 else $\mathrm{F}$ is not XTEA4 
where 148.3564 is the threshold value corresponding to a p-value of $10^{-8}$. The distinguisher will, then, have a false positive probability of $10^{-8}$ and a negligible false negative probability.

\section{$\S 3$ Improved Attack on TEA}

Continuing with our research in the genetic cryptanalysis of TEA, while using essentially the same approach presented in Reference ${ }^{1)}$ and in this paper, we had also tried to find characteristics (bitmasks) that produce a significative deviation of the observed output by XORing them with the inputs, instead of ANDing them as described earlier. This approach strongly resembles what we could call a genetic implementation of the differential attack, ${ }^{9)}$ or a genetic search for impossible differentials in the vein of Reference, ${ }^{6}$ and has lead us to a very interesting result. We have found two characteristics $c_{1}, c_{2}$ in TEA that make that any two keys $k, k^{\prime}$ with the given characteristic $c_{i}$ (i.e. $k \oplus k^{\prime}=c_{i}$ ) produce exactly the same output, no matter how many TEA rounds are used.

These characteristics are:

$$
c_{1}=\{0 x 80000000,0 x 80000000,0 x 00000000,0 x 00000000\}
$$

and

$$
c_{2}=\{0 x 00000000,0 x 00000000,0 x 80000000,0 x 80000000\}
$$

So if we generate any key at random, say

$$
k=\{0 x f 0 e a 8 d 74,0 x e b 4576 c 6,0 x 6 b 14 a b 8 e, 0 x 908 b d 353\}
$$

and we compute

$$
k^{\prime}=k \oplus c_{1}=\{0 x 70 e a 8 d 74,0 x 6 b 4576 c 6,0 x 6 b 14 a b 8 e, 0 x 908 b d 353\}
$$

or

$$
k^{\prime \prime}=k \oplus c_{2}=\{0 x f 0 e a 8 d 74,0 x e b 4576 c 6,0 x e b 14 a b 8 e, 0 x 108 b d 353\}
$$

then for every input block $\mathrm{i}$ and any number of rounds $\mathrm{n}$

$$
T E A_{n}(i, k)=T E A_{n}\left(i, k^{\prime}\right)=T E A_{n}\left(i, k^{\prime \prime}\right)
$$

and, in particular, this happens with the full 32 rounds recommended by the authors.

This is a surprising powerful result, as normally characteristics are discovered for a certain number of rounds and then they vanish exponentially as the number of rounds increases.

\section{$\S 4$ Conclusion}

We have presented a cryptanalytic attack over reduced round versions of the block cipher XTEA based in the new method of constructing distinguishers for cryptographic mappings proposed at Reference. ${ }^{1)}$

We have also shown that XTEA is weak with four or less cycles, that is to say, with 8 rounds or less. This is a result quite similar to those obtained for TEA applying the same genetic cryptanalysis, which forces us to conclude that, 
at least from the genetic cryptanalysis point of view, both TEA and XTEA have a very close security level.

This could also be interpreted as saying that the improvements introduced in XTEA for avoiding the attack presented in Reference ${ }^{5)}$ are limited to make this related-key attack impracticable, but do not improve the overall security of the cipher, which rest very similar to that of TEA.

Although we acknowledge that previous works, specially Reference ${ }^{6)}$ have shown that both TEA and XTEA cannot be considered as secure block ciphers, and have presented stronger attacks on them, we still think the proposed approach remains valuable because it is one of the firsts published attacks using AI techniques that is able of producing worthy cryptanalytic results when confronted against modern ciphers.

Furthermore, nowadays the most powerful attack on these ciphers extend, respectively, to 10 and 12 rounds, and the approach proposed here could be extended to 8 rounds, which makes these results far from trivial and pretty close to the most powerful ones.

Additionally, and regarding the improved attack on TEA presented in this paper, it is, as far as we know, the most powerful attack presented to date against the block cipher.

It is obviously a serious weakness which concrete implications, for example it reduces the effective key-length to 126 bits, and could being straightforwardly used to construct very efficient blackbox distinguishers. This equivalent-keys weakness is not present, at least to the best of our knowledge, in XTEA.

In fact, as one could expect, some minor changes to the TEA round function could avoid it. For example, if we substitute the original line of code (1) by (1') then the problem remains, but when substituting any of them by, for example (2) this weakness, at least thus stated, disappears:

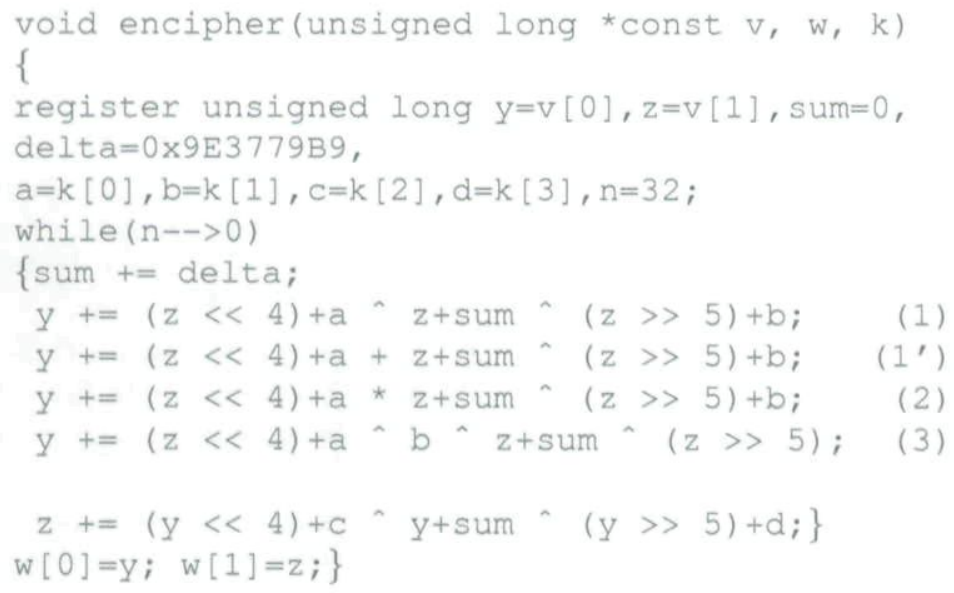

The reason for this is the fact that in 32-bit implementations, operations are made, naturally, $\bmod 2^{32}$ and for that reason, both addition $\bmod 2^{32}$ and xor behave identically on the most significant bit, thus producing the undesirable 
effect of essentially converting (1) in (3) for the leading bit, allowing it to cancelate when simultaneously changing it in $\mathrm{a}=\mathrm{k}[0]$ and $\mathrm{b}=\mathrm{k}[1]$ (or, analogously, at $\mathrm{c}=\mathrm{k}[2]$ and $\mathrm{d}=\mathrm{k}[3])$.

This result is similar to a previously published result ${ }^{5)}$ where the authors presented three attacks on TEA, the first of which is based in simultaneously flipping the next most significant bits (bit 30) of $\mathrm{k}[2]$ and $\mathrm{k}[3]$, the second based in changing the values of both $\mathrm{k}[1]$ (for $k[1] \oplus 2^{31} \oplus 2^{31}$ ) and $\mathrm{v}[1]$ (for $v[1] \oplus 2^{31}$ ). Both attacks, although similar to the proposed one, failed to exhibit full round characteristics.

\section{Acknowledgements}

The work described in this paper was performed as part of the project TIC2002-04498-C05-4 of the Spanish Ministerio de Ciencia y Tecnologia. The authors deeply thank Prof. Andre Seznec of INRIA-IRISA, Rennes, France, and Prof. Caroline Fontaine of LIFL, USTL, Lille, France, for their aid and support, and for carefully reading the manuscript of the paper.

\section{References}

1) Hernandez, J.C. and Isasi, P., "Finding Efficient Distinguishers for Cryptographic Mappings, with an Application to the Block Cipher TEA," Proc. of the 2003 Congress on Evolutionary Computation CEC 2003, pp.341-348, IEEE Press, 2003.

2) Knudsen, L. and Meier, W., "Correlations in RC6 with a Reduced Number of Rounds," Proc. of the Seventh Fast Software Encryption Workshop, Springer-Verlag, 2000.

3) Shimoyama, T., Takeuchi, K. and Hayakawa, J., "Correlation Attack to the Block Cipher RC5 and the Simplified Variants of RC6," Proc. of the Third AES Candidate Conference AES3, 2000.

4) Wheeler, D. and Needham, R., "TEA, A Tiny Encryption Algorithm," Proc. of the 1995 Fast Software Encryption Workshop, pp. 97-110, Springer-Verlag. 1995.

5) Wagner, D., Kelsey, J. and Schneier, B., "Related-Key Cryptoanalysis of 3WAY, Biham-DES, CAST, DES-X, NewDES, RC2 and TEA," Proc. of the ICICS'97 Conference, pp. 233-246, Springer-Verlag, 1997.

6) Moon, D., Kyungdeok, H. and Wonil, L., et al., "Impossible Differential Cryptanalysis of Reduced Round XTEA and TEA," Proc. of the Fast Software Encryption, FSE 2002, Springer LNCS, 2365, pp. 49-60, Leuven, Belgium, February 4-6, 2002.

7) Kelsey, J., Schneier, B. and Wagner, D., "Mod $n$ Cryptoanalysis with Applications against RC5P and M6," Proc. of the 1999 Fast Software Encryption Workshop, pp. 139-155, Springer-Verlag, 1999.

8) Forre, R., "The Strict Avalanche Criterion: Special Properties of Boolean Functions and Extended Definition," Advances in Cryptology - Proc. of CRYPTO'88, LNCS, 403, pp.450-468, Springer-Verlag, 1988. 
9) Biham, E., Biryukov, A. and Shamir, A., "Cryptanalysis of Skipjack Reduced to 31 rounds Using Impossible Differentials," Technion Computer Science Department Technical Report CS0947-1998.

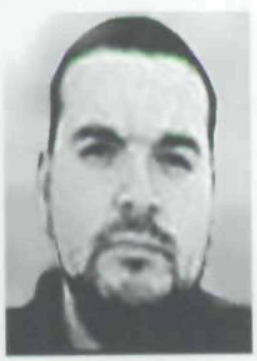

Julio César Hernández Castro, Ph.D.: He received Graduate Degree in Mathematics at Complutense University, M.Sc. in Coding Theory and Network Security at Valladolid University, and a Ph.D. in Computer Science at Carlos III University. His current research is fundamentally oriented to find and develop applications of techniques of artificial intelligence to cryptography and computer security. He has published more than 50 papers in cryptography, cryptanalysis and network security at International Journals and Conferences. He loves chess and go. At this moment, he is a Postdoctoral researcher at LIFL, Universite de Lille I, France.

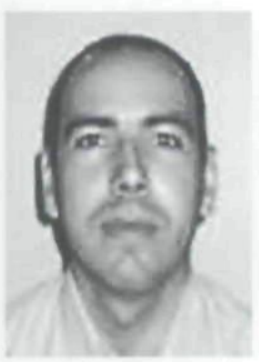

Pedro Isasi Viñuela: He received Graduate and Doctor in Computer science by the Polytechnical University of Madrid in 1994. Currently he is Professor of Computational Sciences and Artificial Intelligence in the Department of Computer Science in Carlos III University. His research is focused on the field of Artificial intelligence, on problems of Optimization and automatic learning, in evolutionary systems and in artificial neural networks. He has published two books in the area. 